\title{
On the exact asymptotics for the stationary sojourn time distribution in a tandem of queues with light-tailed service times *
}

\author{
S.G. Foss \\ Heriot-Watt University, Edinburgh and \\ Institute of Mathematics, Novosibirsk
}

\begin{abstract}
We study the asymptotics of the stationary sojourn time $Z$ of a "typical customer" in a tandem of single-server queues. It is shown that, in a certain "intermediate" region of light-tailed service time distributions, $Z$ may take a large value mostly due to a large value of a single service time of one of customers. Arguments used in the paper allow us to obtain also an elementary proof of the logarithmic asymptotics for the tail distribution of the stationary sojourn time in the whole class of light-tailed distributions.
\end{abstract}

Keywords: tandem of queues, sojourn time, large deviations, Cramer condition, exact and logarithmic asymptotics, class $\mathcal{S}_{\gamma}$.

\section{Introduction and main results}

Consider an open queueing network which is a tandem of two single-server queues $G I / G I / 1 \rightarrow$ $/ G I / 1$ with "first-come-first-served" service disciplines.

Consider three mutually independent sequences of non-negative random variables $\left\{\tau_{n}\right\}$, $\left\{\sigma_{n}^{(1)}\right\}$ and $\left\{\sigma_{n}^{(2)}\right\}$, each of which is an i.i.d. sequence. Here $\tau_{n}$ is the inter-arrival time between the $(n-1)$ st and $n$th customers (with mean $a=\mathbf{E} \tau_{1}$ ). Customer $n$ receives service in the first queue of duration $\sigma_{n}^{(1)}$ ( with distribution function $G^{(1)}$ and positive mean $b^{(1)}=$ $\mathbf{E} \sigma_{1}^{(1)}$ ) and then in the second of duration $\sigma_{n}^{(2)}$ (with distribution function $G^{(2)}$ and positive mean $\left.b^{(2)}=\mathbf{E} \sigma_{1}^{(2)}\right)$. Is it assumed that the network is stable, i.e. $\max \left(b^{(1)}, b^{(2)}\right)<a$. It is well-known (see, for example, [1]) that, under this assumption, there exists a unique stationary (limiting) distribution of the sojourn time $Z$ in the network (i.e. of the duration of time between a customer's arrival to the first queue and its departure from the second queue) and, for any initial condition, the distribution of the sojourn time $Z_{n}$ of customer $n$ converges in the total variation norm to the limiting distribution, as $n \rightarrow \infty$.

The following representation is also known (see, e.g., [2]):

$$
Z=\sup _{0 \leq n \leq m<\infty}\left(\sum_{-m}^{-n} \sigma_{j}^{(1)}+\sum_{-n}^{0} \sigma_{j}^{(2)}-\sum_{-m}^{-1} \tau_{j}\right) .
$$

One may interpret formula (11) as follows. Assume that the network has been working for an infinitely long time, starting from time $-\infty$. Then $Z=Z_{0}$ is the sojourn time of customer

\footnotetext{
${ }^{*}$ The research was partially supported by the EPSRC Grant EP/E033717/1
} 
0 that arrives at time instant $t=0$. Note that $Z$ is a monotone function of all variables in the right-hand side of (1): it monotonically increases with the growth of any of the $\sigma$ 's and with the decrease of any of the $\tau$ 's.

The paper deals with a study of the asymptotics of probability $\mathbf{P}(Z>x)$ when $x$ tends to infinity. We consider the case where service times distributions in both queues are lighttailed, i.e.

$$
\varphi_{\sigma_{1}^{(i)}}(\lambda)<\infty
$$

for $i=1,2$ and for some positive $\lambda$. Here we use the standard notation: $\varphi_{X}(\lambda)=\mathbf{E} e^{\lambda X}$ is the exponential moment of a random variable $X$ at point $\lambda$. For short, we write in the sequel $\varphi_{(i)}(\lambda)=\varphi_{\sigma_{1}^{(i)}}(\lambda)$ for $i=1,2$ and $\varphi_{\tau}(\lambda)=\varphi_{\tau_{1}}(\lambda)$. For $i=1,2$, denote

$$
\gamma^{(i)}=\sup \left\{\lambda: \varphi_{(i)}(\lambda) \varphi_{\tau}(-\lambda) \leq 1\right\} \in(0, \infty)
$$

and let

$$
\gamma=\min \left(\gamma^{(1)}, \gamma^{(2)}\right)
$$

For two positive functions $f_{1}$ and $f_{2}$ and for a constant $d \geq 0$, the notation $f_{1}(x) \sim d f_{2}(x)$ means that $f_{1}(x) / f_{2}(x) \rightarrow d$ as $x \rightarrow \infty$. In particular, if $d=0$, then $f_{1}(x)=o\left(f_{2}(x)\right)$.

The following logarithmic ("rough") asymptotics holds (see, for example, [3, 4]):

Theorem 1. Under condition (2),

$$
-\ln \mathbf{P}(Z>x) \sim \gamma x
$$

All (known to us) proofs of Theorem 1 (see, for example, [3, 4]) use the techniques of large deviations.

Our main result (Theorem 2) provides the exact asymptotics of large deviations, under the following additional assumption:

$$
R \equiv \max \left(\varphi_{(1)}(\gamma), \varphi_{(2)}(\gamma)\right) \varphi_{\tau}(-\gamma)<1
$$

In particular, (3) implies the finiteness of $\mathbf{E} e^{\gamma Z}$. Indeed,

$$
\mathbf{E} e^{\gamma Z} \leq \sum_{0 \leq n \leq m} \mathbf{E} \exp \left(\gamma\left(\sum_{-m}^{-n} \sigma_{j}^{(1)}+\sum_{-n}^{0} \sigma_{j}^{(2)}-\sum_{-m}^{-1} \tau_{j}\right)\right) \leq(1-R)^{-2} \varphi_{\tau}^{-2}(-\gamma)<\infty .
$$

In order to state Theorem 2 , we need a number of further definitions and notation.

We use the same symbol $F$ to denote a probability distribution on the real line and also its distribution function. Let $\bar{F}$ be the tail of distribution $F$, i.e.. $\bar{F}(x)=1-F(x)$, and $F^{* n}$ the $n$-fold convolution of $F$. A distribution function $F$ belongs to the class $\mathcal{L}_{\beta}, \beta \geq 0$ if

$$
\bar{F}(x)>0 \text { for all } x \quad \text { and } \lim _{x \rightarrow \infty} \frac{\bar{F}(x-y)}{\bar{F}(x)}=e^{\beta y} \quad \text { for any fixed } y .
$$

Due to the monotonicity of $\bar{F}$, the convergence in (5) is necessarily uniform in $y$ on any compact set. Therefore we may find such a function $h(x) \uparrow \infty, h(x)=o(x)$ that

$$
\lim _{x \rightarrow \infty} \sup _{|y| \leq h(x)}\left|\frac{\bar{F}(x+y)}{\bar{F}(x)} e^{\beta y}-1\right|=0 .
$$


If $h_{1}$ and $h_{2}$ are two functions satisfying (6), then the function $h_{3}(x)=h_{1}(x)+h_{2}\left(x-h_{1}(x)\right)$ has the same property.

The distribution function $F$ of a random variable $X$ belongs to the class $\mathcal{S}_{\beta}, \beta \geq 0$, if $F \in \mathcal{L}_{\beta}, \varphi_{X}(\beta)<\infty$ and

$$
\overline{F^{* 2}}(x)=\mathbf{P}\left(X_{1}+X_{2}>x\right) \sim 2 \varphi_{X}(\beta) \bar{F}(x) \quad \text { as } x \rightarrow \infty
$$

where $X_{1}$ and $X_{2}$ are two independent copies of $X$. Here, with necessity,

$$
\begin{aligned}
\mathbf{P}\left(X_{1}+X_{2}>x\right) & \sim \mathbf{P}\left(X_{1}+X_{2}>x, X_{1} \leq h(x)\right)+\mathbf{P}\left(X_{1}+X_{2}>x, X_{2} \leq h(x)\right) \\
& \sim \mathbf{P}\left(X_{1}+X_{2}>x, X_{1} \geq x-h(x)\right)+\mathbf{P}\left(X_{1}+X_{2}>x, X_{2} \geq x-h(x)\right)
\end{aligned}
$$

where $h(x)$ is any function satisfying condition (6) (see, for example, [5]).

A typical example of a distribution $F \in \mathcal{S}_{\beta}$ with $\beta>0$ is a distribution with the tail $\bar{F}(x)=C x^{-\alpha} e^{-\beta x}$ for some $\alpha>1, C \in(0,1]$ and all $x \geq 1$.

Theorem 2. Assume that condition (3) holds. Suppose that

$$
\overline{G^{(i)}}(x) \sim c^{(i)} \bar{F}(x), \quad i=1,2
$$

for some function $F \in \mathcal{S}_{\gamma}$ and constants $c^{(1)} \geq 0$ and $c^{(2)} \geq 0$. Then

$$
\mathbf{P}(Z>x) \sim \sum_{i=1}^{2} \sum_{j=-\infty}^{0} \mathbf{P}\left(Z>x, \sigma_{j}^{(i)}>x-h(x)\right) \sim K \bar{F}(x)
$$

where $h(x)$ is any function satisfying conditions (6) and the constant $K$ is determined below in formula (25).

Remark 1. Since the exact representation of constant $K$ is complex and depends on characteristics which are not "computable", it seems to be reasonable to provide useful upper and lower bounds for $K$. For that, let, for $i=1,2, R_{i}=\varphi_{(i)}(\gamma) \varphi_{\tau}(-\gamma)$ and $R=\max \left(R_{1}, R_{2}\right)$. Then

$$
\frac{c^{(1)} \varphi_{(2)}(\gamma)}{1-R}+\frac{c^{(2)} \varphi_{(1)}(\gamma)}{1-R_{2}} \leq K \leq \frac{1}{\left(1-R_{1}\right)\left(1-R_{2}\right)}\left(\frac{c^{(1)} \varphi_{(2)}(\gamma)}{1-R_{1}}+\frac{c^{(2)} \varphi_{(1)}(\gamma)}{1-R_{2}}\right) .
$$

Remark 2. The coefficients $c^{(1)}$ and $c^{(2)}$ in the statement of Theorem 2 may be either positive or zero. If both coefficients are positive, then, with necessity, $\gamma^{(1)}=\gamma^{(2)}=\gamma$ and - as it follows from Property 1 (see Appendix) - both distributions $G^{(i)}, i=1,2$, have to belong to the class $\mathcal{S}_{\gamma}$. If only one of the coefficients is positive, say if $c^{(1)}>0$ and $c^{(2)}=0$, then the distribution $G^{(1)}$ belongs to the class $\mathcal{S}_{\gamma}$ and $\gamma^{(2)} \geq \gamma^{(1)}=\gamma$. Finally, if $c^{(1)}=c^{(2)}=0$, then also $K=0$, as it follows from (10).

Remark 3. Taking into account the monotonicity properties (see the comments after formula (1), we can obtain the following "one-side" analogues of Theorem 2.

If, in the statement of Theorem 2, one replaces condition (8) by $\lim \sup _{x \rightarrow \infty} \overline{G^{(i)}}(x) / \bar{F}(x) \leq$ $c^{(i)}, i=1,2$ (or by $\left.\liminf _{x \rightarrow \infty} \overline{G^{(i)}}(x) / \bar{F}(x) \geq c^{(i)}, i=1,2\right)$, then the following holds: $\limsup _{x \rightarrow \infty} \mathbf{P}(Z>x) / \bar{F}(x) \leq K$ (or, respectively, $\left.\liminf _{x \rightarrow \infty} \mathbf{P}(Z>x) / \bar{F}(x) \geq K\right)$, with the same $K$.

Remark 4. A natural analogue of Theorem 2 holds for tandems of any finite number of queues and, more generally, for tree-like queueing networks. However, an explicit representation of the constant $K$ and even its bounds become less and less tractable as $n$ increases.

Therefore we decided to consider the case $n=2$ only. 
Remark 5. The approach in the first part of the proof of Theorem 2 (i.e. the construction of upper and lower bounds) allows us also to obtain a simple proof of Theorem 1 without use of the techiques of the large deviations theory (see Subsection 3.2).

Remark 6. The proposed method of proof is based on ideas developed in [6] and applied therein to obtaining the distributional asymptotics for $\mathbf{P}(Z>x)$ in tandems of queues with subexponential service times distributions (which are heavy-tailed). Also, in [6], the asymptotics for the stationary waiting time in the second queue were obtained. Similar asymptotics may be found under the conditions of this paper, but by the use of essentially more complicated formulae.

Remark 7 . In addition to the asymptotics for the tail $\mathbf{P}(Z>x), x \rightarrow \infty$, one can use results from [5] to obtain the asymptotics for prestationary probabilities $\mathbf{P}\left(Z_{k}>x\right)$ (which are uniform in $k$ ). Here

$$
Z_{k}=\sup _{0 \leq n \leq m \leq k}\left(\sum_{-m}^{-n} \sigma_{j}^{(1)}+\sum_{-n}^{0} \sigma_{j}^{(2)}-\sum_{-m}^{-1} \tau_{j}\right) .
$$

A proof of Theorem 2 is given in the next Section. Section 3 contains a useful auxiliary information about the class $\mathcal{S}_{\beta}$, a simple proof of Theorem 1 and some further comments.

\section{Proof}

\subsection{Upper and lower bounds for the stationary sojourn time}

Lower bound. For $i=1,2$, let

$$
Z^{(i)}=\sigma_{0}^{(i)}+\max _{n \geq 0} \sum_{-n}^{-1}\left(\sigma_{j}^{(i)}-\tau_{j}\right)
$$

(where $\sum_{0}^{-1}=0$ ). Then $Z^{(1)}=\sigma_{0}^{(1)}+W^{(1)}$ where $W^{(1)}=\max _{n \geq 0} \sum_{-n}^{-1}\left(\sigma_{j}^{(1)}-\tau_{j}\right)$. Here $Z^{(1)}$ (respectively, $W^{(1)}$ ) is the stationary sojourn (respectively, waiting) time in the first queue. However, it is more convenient to propose a slightly different interpretation of the formula above: $Z^{(1)}$ (respectively, $W^{(1)}$ ) is the stationary sojourn (respectively, waiting) time in an auxiliary tandem of queues where all service times in the second queue are equal to zero. Similarly, $Z^{(2)}=\sigma_{0}^{(2)}+W^{(2)}$ with $W^{(2)}=\max _{n \geq 0} \sum_{-n}^{-1}\left(\sigma_{j}^{(2)}-\tau_{j}\right)$, and $Z^{(2)}$ (respectively, $W^{(2)}$ ) is the stationary sojourn (respectively, waiting) time in an auxiliary tandem of queues where all service times in the first queue and replaced by zero.

The monotonicity of $Z$ in all variables in (1) implies the following bound

$$
Z \geq \max \left(Z^{(1)}, Z^{(2)}\right) \quad \text { a.s. }
$$

and, in particular,

$$
\mathbf{P}(Z>x) \geq \max \left(\mathbf{P}\left(Z^{(1)}>x\right), \quad \mathbf{P}\left(Z^{(2)}>x\right)\right) .
$$

Upper bound. Let $L \geq 1$ be an integer. Introduce an auxiliary single-server queue with i.i.d. inter-arrival times $\widetilde{\tau}_{n}$ and (independent of them) i.i.d. service times $\widetilde{\sigma}_{n}$ where

$$
\widetilde{\tau}_{n}=\sum_{1}^{L} \tau_{(n-1) L+i} \quad \text { and } \quad \tilde{\sigma}_{n}=\max _{1 \leq j \leq L}\left(\sum_{i=1}^{j} \sigma_{(n-1) L+i}^{(1)}+\sum_{i=j}^{L} \sigma_{(n-1) L+i}^{(2)}\right) .
$$


Here the random variable $\widetilde{\sigma}_{1}$ may be viewed as follows. Assume that customers $1, \ldots, L$ arrive simultaneously at time instant $t=0$ into an empty network. Then $\widetilde{\sigma}_{1}$ is the time of the last completion of service of these customers in the second queue. It is not difficult to prove (see, for example, [7]) that $\mathbf{E} \widetilde{\sigma}_{1} / L \rightarrow \max \left(b^{(1)}, b^{(2)}\right)$ as $L \rightarrow \infty$. Hence $\mathbf{E} \widetilde{\sigma}_{1}<\mathbf{E} \widetilde{\tau}_{1}$ for all sufficiently large $L$. We fix such an $L$ and define

$$
\widetilde{W}=\max _{n \geq 0} \sum_{-n}^{-1}\left(\widetilde{\sigma}_{n}-\widetilde{\tau}_{n}\right)<\infty \quad \text { a.s. }
$$

(respectively $\widetilde{Z}=\widetilde{\sigma}_{0}+\widetilde{W}$ ), the stationary sojourn (respectively waiting) time of customer 0 in this queueing system, which is a.s. finite. The monotonicity properties of $Z$ imply (see, for example, [7, 6]) that

$$
Z \leq \widetilde{Z} \quad \text { a.s. }
$$

\subsection{Proof of Theorem 2 ,}

We prove Theorem 2 only for $c^{(1)}+c^{(2)}>0$, the statement in the case $c^{(1)}=c^{(2)}=0$ follows by monotonicity.

$>$ From [5, Theorem 1] and from inequality (13), we get

$$
\liminf _{x \rightarrow \infty} \frac{\mathbf{P}(Z>x)}{\bar{F}(x)} \geq \max \left(c^{(1)}, c^{(2)}\right) .
$$

Now we could use (14) and obtain the upper bound

$$
\limsup _{x \rightarrow \infty} \frac{\mathbf{P}(Z>x)}{\bar{F}(x)} \leq K_{0},
$$

for some positive constant $K_{0}$. However, we need an explicit representation for events leading to large values of $Z$. For that, we find it convenient to work with a more "rough" upper bound than (14). Namely, take an arbitrary positive number $T>0$ and define random variables $\widehat{\sigma}_{n}$ by the equalities

$$
\widehat{\sigma}_{n}=\Sigma_{n} \mathbf{I}\left(\Sigma_{n}>T\right)+\widetilde{\sigma}_{n} \mathbf{I}\left(\Sigma_{n} \leq T\right)
$$

where

$$
\Sigma_{n}=\sum_{i=1}^{L}\left(\sigma_{(n-1) L+i}^{(1)}+\sigma_{(n-1) L+i}^{(2)}\right) \geq \widetilde{\sigma}_{n}
$$

and $\mathbf{I}$ is the indicator function. Clearly, $\widehat{\sigma}_{n} \geq \widetilde{\sigma}_{n}$ a.s. Further, due to Properties 1 and 2 from the Appendix, the common distribution function of the random variables $\widehat{\sigma}_{n}$ belongs to the class $\mathcal{S}_{\gamma}$, and $\mathbf{P}\left(\widehat{\sigma}_{1}>x\right) \sim C \bar{F}(x)$ for some positive $C$. Indeed, the distribution of $\Sigma_{n}$ belongs to the class $\mathcal{S}_{\gamma}$ by Property 2 . Since, for any $x>T, \widehat{\sigma}_{n}>x$ if and only if $\Sigma_{n}>x$, we conclude that $\mathbf{P}\left(\widehat{\sigma}_{n}>x\right) \sim \mathbf{P}\left(\Sigma_{n}>x\right)$ as $x \rightarrow \infty$ and, therefore, the distribution $\widehat{\sigma}_{n}$ also belongs to the class $\mathcal{S}_{\gamma}$, due to Property 1 ..

We know that $\mathbf{E} \widetilde{\sigma}<\mathbf{E} \widetilde{\tau}_{1}=L \mathbf{E} \tau_{1}$ for all sufficiently large $L$. Also, $\widetilde{\varphi}(\gamma)=\mathbf{E} e^{\gamma \widetilde{\sigma}_{1}}<$ $1 / \mathbf{E} e^{-\gamma \widetilde{\tau}_{1}}$ for all large $L$, from (3) and (28). Choose such an $L$. Further, $\mathbf{E} \widehat{\sigma} \rightarrow \mathbf{E} \widetilde{\sigma}$ and $\mathbf{E} e^{\gamma \widehat{\sigma}_{1}} \rightarrow \mathbf{E} e^{\gamma \widetilde{\sigma}_{1}}$ as $T \rightarrow \infty$. Therefore we can take a sufficiently large $T$ for the inequalities

$$
\mathbf{E} \widehat{\sigma}_{1}<L \mathbf{E} \tau_{1} \quad \text { and } \quad \mathbf{E} e^{\gamma \widehat{\sigma}_{1}}<1 / \mathbf{E} e^{-\gamma \widetilde{\tau}_{1}}
$$


to hold. Then the single-server queueing system with i.i.d. inter-arrival times $\widetilde{\tau}_{n}$ (see Subsection 2.1 for the definition) and service times $\widehat{\sigma}_{n}$ is stable. Let $\widehat{W}$ denote the stationary waiting time in this system,

$$
\widehat{W}=\max _{n \geq 0} \sum_{-n}^{-1}\left(\widehat{\sigma}_{n}-\widetilde{\tau}_{n}\right)<\infty \quad \text { a.s. }
$$

Then $\widehat{W}$ coincides in distribution with the supremum of a random walk with increments $\widehat{\sigma}_{n}-\widetilde{\tau}_{n}$. By Property 4 from the Appendix and from (17), $\mathbf{P}(\widehat{W}>x) \sim C \bar{F}(x)$ for some $C>0$ and, by Property 2, the distribution of the random variable $\widehat{W}$ belongs to the class $\mathcal{S}_{\gamma}$. From the monotonicity properties,

$$
Z \leq \widehat{Z} \equiv \widehat{W}+\sigma_{0}^{(1)}+\sigma_{0}^{(2)}
$$

where the increments in the right-hand side are mutually independent, and the tail distribution of each of them is asymptotically equivalent to $\bar{F}(x)$, up to a multiplicative non-negative constant (where at least one of these constants is strictly positive). By Property 2 from the Appendix, the distribution of $\widehat{Z}$ also belongs to the class $\mathcal{S}_{\gamma}$ and

$$
\begin{aligned}
\mathbf{P}(\widehat{Z}>x) & =\mathbf{P}\left(\bigcup_{i=1}^{2}\left\{\widehat{Z}>x, \sigma_{0}^{(i)}>x-h_{1}(x)\right\} \bigcup\left\{\widehat{Z}>x, \widehat{W}>x-h_{1}(x)\right\}\right)+o(\bar{F}(x)) \\
& =\sum_{i=1}^{2} \mathbf{P}\left(\widehat{Z}>x, \sigma_{0}^{(i)}>x-h_{1}(x)\right)+\mathbf{P}\left(\widehat{Z}>x, \widehat{W}>x-h_{1}(x)\right)+o(\bar{F}(x))
\end{aligned}
$$

for any function $h_{1}$ satisfying (6). Note that if $h_{1}$ and $h$ are two such functions, then

$$
\mathbf{P}\left(\widehat{Z}>x, \sigma_{0}^{(i)}>x-h_{1}(x)\right)=\mathbf{P}\left(\widehat{Z}>x, \sigma_{0}^{(i)}>x-h(x)\right)+o(\bar{F}(x)) .
$$

Make use of the following simple relations. Let $A, B$ and $C$ be three events. If

$$
\mathbf{P}(A)=\mathbf{P}(A \cap B)+v,
$$

then

$$
\mathbf{P}(A \cap C)=\mathbf{P}(A \cap C \cap B)+\widehat{v}
$$

where $0 \leq \widehat{v} \leq v$. In particular, if $C \subseteq A$, then the last equality in transformed into

$$
\mathbf{P}(C)=\mathbf{P}(C \cap B)+\widehat{v} .
$$

Applying this to the events $A=\{\widehat{Z}>x\}$ and $C=\{Z>x\}$, we arrive at the equation

$$
\mathbf{P}(Z>x)=\sum_{i=1}^{2} \mathbf{P}\left(Z>x, \sigma_{0}^{(i)} \geq x-h_{1}(x)\right)+\mathbf{P}\left(Z>x, \widehat{W}>x-h_{1}(x)\right)+o(\bar{F}(x)) .
$$

By Property 4 from the Appendix, for any $\varepsilon>0$, there exists a sufficiently large $N$ such that

$$
\begin{aligned}
\mathbf{P}\left(\widehat{W}>x-h_{1}(x)\right) & =\mathbf{P}\left(\bigcup_{j=1}^{N}\left\{\widehat{W}>x-h_{1}(x), \widehat{\sigma}_{-j}-\tau_{-j}>x-h_{1}(x)-h_{2}\left(x-h_{1}(x)\right)\right\}\right)+g_{1}(x) \\
& =\mathbf{P}\left(\bigcup_{j=1}^{N}\left\{\widehat{W}>x-h_{1}(x), \widehat{\sigma}_{-j}>x-h_{1}(x)-h_{2}\left(x-h_{1}(x)\right)\right\}\right)+g_{2}(x) \\
& =\sum_{j=1}^{N} \mathbf{P}\left(\widehat{W}>x-h_{1}(x), \widehat{\sigma}_{-j}>x-h_{1}(x)-h_{2}\left(x-h_{1}(x)\right)\right)+g_{3}(x)
\end{aligned}
$$


where $h_{2}$ is any function satisfying (6) and $0 \leq g_{i}(x) \leq \varepsilon \bar{F}(x)+o(\bar{F}(x))$ for $i=1,2,3$. The latter inequality means that $\lim _{\sup } \rightarrow \infty g_{i}(x) / \bar{F}(x) \leq \varepsilon$.

Using again (20), this time with $A=\left\{\widehat{W}>x-h_{1}(x)\right\}$ and $C=\{Z>x\}$, we get

$\left.\mathbf{P}\left(Z>x, \widehat{W}>x-h_{1}(x)\right)=\sum_{j=1}^{N} \mathbf{P}\left(Z>x, \widehat{W}>x-h_{1}(x)\right), \widehat{\sigma}_{-j}>x-h_{1}(x)-h_{2}\left(x-h_{1}(x)\right)\right)+g_{4}(x)$

where $0 \leq g_{4}(x) \leq \varepsilon \bar{F}(x)+o(\bar{F}(x))$.

By choosing an appropriate $h_{2}$, the right-hand side of the latter inequality may be made simpler, by the exclusion the inequality $\left\{\widehat{W}>x-h_{1}(x)\right\}$. Indeed, put

$$
\left.P(x)=\mathbf{P}\left(Z>x, \widehat{W} \leq x-h_{1}(x)\right), \widehat{\sigma}_{-j}>x-h_{1}(x)-h_{2}\left(x-h_{1}(x)\right)\right)
$$

and note that

$$
\begin{aligned}
P(x) & \left.\leq \mathbf{P}\left(\widehat{Z}>x, \widehat{W} \leq x-h_{1}(x)\right), \widehat{\sigma}_{-j}>x-h_{1}(x)-h_{2}\left(x-h_{1}(x)\right)\right) \\
& \leq \mathbf{P}\left(\sigma_{0}^{(1)}+\sigma_{0}^{(2)}>h_{1}(x), \widehat{\sigma}_{-j}>x-h_{1}(x)-h_{2}\left(x-h_{1}(x)\right)\right) \\
& =(1+o(1))\left(c^{(1)}+c^{(2)}\right) \bar{F}\left(h_{1}(x)\right) \cdot C \bar{F}\left(x-h_{1}(x)-h_{2}\left(x-h_{1}(x)\right)\right) \\
& =(1+o(1)) \widehat{C} e^{\gamma\left(h_{1}(x)+h_{2}\left(x-h_{1}(x)\right)\right)} \bar{F}\left(h_{1}(x)\right) \bar{F}(x)
\end{aligned}
$$

where the last equality follows from the remark after formula (6) and $\widehat{C}=C\left(c^{(1)}+c^{(2)}\right)$. From $\int_{0}^{\infty} e^{\gamma t} d F(t)<\infty$, we get $e^{\gamma t} \bar{F}(t) \rightarrow 0$ as $t \rightarrow \infty$ and, therefore, $\bar{F}\left(h_{1}(x)\right) e^{\gamma h_{1}(x)} \rightarrow 0$ as $x \rightarrow \infty$. Thus, we may take $h_{2}$ so slowly increasing to infinity, that $\bar{F}\left(h_{1}(x)\right) e^{\gamma\left(h_{1}(x)+h_{2}\left(x-h_{1}(x)\right)\right)}$ also tends to zero. Then $P(x)=o(\bar{F}(x))$ and, therefore,

$$
\mathbf{P}\left(Z>x, \widehat{W}>x-h_{1}(x)\right)=\sum_{j=1}^{N} \mathbf{P}\left(Z>x, \widehat{\sigma}_{-j}>x-h_{3}(x)\right)+g_{5}(x)
$$

where $0 \leq g_{5}(x) \leq \varepsilon \bar{F}(x)+o(\bar{F}(x))$ and the function $h_{3}(x)=h_{1}(x)+h_{2}\left(x-h_{1}(x)\right)$ satisfies (6).

For all sufficiently large $x$, the events $\left.\left\{\widehat{\sigma}_{-j}>x-h_{3}(x)\right)\right\}$ and $\left.\left\{\Sigma_{-j}>x-h_{3}(x)\right)\right\}$ either occur or do not occur simultaneously. Therefore $\mathbf{P}\left(Z>x, \widehat{\sigma}_{-j}>x-h_{3}(x)\right)=\mathbf{P}(Z>$ $\left.x, \Sigma_{-j}>x-h_{3}(x)\right)$. Applying Property 2 from the Appendix to the random variables $\Sigma_{-j}$, we get

$$
\begin{aligned}
\mathbf{P}\left(Z>x, \widehat{\sigma}_{-j}>x-h_{3}(x)\right) & =\sum_{i=1}^{2} \sum_{l=(j-1) L+1}^{j L} \mathbf{P}\left(Z>x, \widehat{\sigma}_{-j}>x-h_{3}(x), \sigma_{-l}^{(i)}>x-h_{3}(x)-h_{4}\left(x-h_{3}(x)\right)\right) \\
& +o(\bar{F}(x))
\end{aligned}
$$

where $h_{4}$ is any function satisfying (6). Assuming that $h_{4}$ satisfies an extra condition (which is analogues to that on $h_{2}$ ), we may exclude the inequality $\left\{\widehat{\sigma}_{-j}>x-h_{3}(x)\right\}$ from the right-hand side of the last relation. Letting $h(x)=h_{3}(x)+h_{4}\left(x-h_{3}(x)\right)$, we arrive at the equality

$$
\mathbf{P}\left(Z>x, \widehat{\sigma}_{-j}>x-h_{3}(x)\right)=\sum_{i=1}^{2} \sum_{l=(j-1) L+1}^{j L} \mathbf{P}\left(Z>x, \sigma_{-l}^{(i)}>x-h(x)\right)+o(\bar{F}(x)) .
$$

Substituting the relations obtained into formula (21) and using (19), we get finally:

$$
\mathbf{P}(Z>x)=\sum_{i=1}^{2} \sum_{j=0}^{N L} \mathbf{P}\left(Z>x, \sigma_{-j}^{(i)}>x-h(x)\right)+g(x)
$$


where $0 \leq g(x) \leq \varepsilon \bar{F}(x)+o(\bar{F}(x))$.

Now we study the asymptotics of each individual summand in the double sum in the right-hand side of (22). Let

$$
W_{j}^{(1)}=\max \left(0, \sup _{n \geq 1} \sum_{i=1}^{n}\left(\sigma_{-j-i}^{(1)}-\tau_{-j-i}\right)\right)
$$

and notice that the distribution of $W_{j}^{(1)}$ does not depend on $j$. Further, let

$$
Y_{j}^{(1)}=\max _{0 \leq k \leq-j}\left(\sum_{i=-j+1}^{-j+k} \sigma_{i}^{(1)}+\sum_{i=-j+k}^{0} \sigma_{i}^{(2)}\right)
$$

and

$V_{j}^{(1)}=\max \left(\sup _{-j<n \leq m<\infty}\left(\sum_{-m}^{-n} \sigma_{i}^{(1)}+\sum_{-n}^{0} \sigma_{i}^{(2)}-\sum_{-m}^{-1} \tau_{i}\right), \max _{0 \leq n \leq m<-j}\left(\sum_{-m}^{-n} \sigma_{i}^{(1)}+\sum_{-n}^{0} \sigma_{i}^{(2)}-\sum_{-m}^{-1} \tau_{i}\right)\right)$.

Then, for any $j \geq 0$,

$$
Z=\max \left(V_{j}^{(1)}, W_{j}^{(1)}+\sigma_{-j}^{(1)}+Y_{j}^{(1)}-\sum_{i=-j}^{-1} \tau_{i}\right)
$$

where the random variables $\left(W_{j}^{(1)}, Y_{j}^{(1)}, V_{j}^{(1)}, \sum_{-j}^{-1} \tau_{i}\right)$ mutually do not depend on $\sigma_{-j}^{(1)}$. Put $Q_{j}^{(1)}=W_{j}^{(1)}+Y_{j}^{(1)}-\sum_{-j}^{-1} \tau_{i}$. For any $j=0, \ldots, N L$,

$$
\begin{aligned}
\mathbf{P}\left(Z>x, \sigma_{-j}^{(1)}>x-h(x)\right) & =\mathbf{P}\left(Q_{j}^{(1)}+\sigma_{-j}^{(1)}>x, \sigma_{-j}^{(1)}>x-h(x)\right)+o(\mathbf{P}(Z>x)) \\
& =\int_{0}^{h(x)} \mathbf{P}\left(Q_{j}^{(1)} \in d t\right) \mathbf{P}\left(\sigma_{-j}^{(1)}>x-t\right)+o(\mathbf{P}(Z>x))+o(\bar{F}(x)) \\
& =c^{(1)} \bar{F}(x) \int_{0}^{h(x)} \mathbf{P}\left(Q_{j}^{(1)} \in d t\right) e^{-\gamma t}+o(\mathbf{P}(Z>x)+\bar{F}(x)) \\
& =c^{(1)} \bar{F}(x) \mathbf{E} e^{\gamma W_{0}^{(1)}} \mathbf{E} e^{\gamma Y_{j}^{(1)}}\left(\varphi_{\tau}(-\gamma)\right)^{j}+o(\mathbf{P}(Z>x)+\bar{F}(x))
\end{aligned}
$$

We clarify now each of four equalities above. The first of them follows from

$$
\begin{aligned}
\mathbf{P}\left(Z>x, \sigma_{-j}^{(1)}>x-h(x)\right) & =\mathbf{P}\left(Q_{j}^{(1)}+\sigma_{-j}^{(1)}>x, \sigma_{-j}^{(1)}>x-h(x)\right) \\
& +\mathbf{P}\left(V_{j}^{(1)}>x, Q_{j}^{(1)}+\sigma_{-j}^{(1)} \leq x, \sigma_{-j}^{(1)}>x-h(x)\right)
\end{aligned}
$$

where the second summand is not bigger than

$$
\begin{aligned}
\mathbf{P}\left(V_{j}^{(1)}>x, \sigma_{-j}^{(1)}>x-h(x)\right) & =\mathbf{P}\left(V_{j}^{(1)}>x\right) \mathbf{P}\left(\sigma_{-j}^{(1)}>x-h(x)\right) \\
& \leq \mathbf{P}(Z>x) \mathbf{P}\left(\sigma_{-j}^{(1)}>x-h(x)\right)=o(\mathbf{P}(Z>x)) .
\end{aligned}
$$

Further,

$$
\begin{aligned}
\mathbf{P}\left(Q_{j}^{(1)}+\sigma_{-j}^{(1)}>x, \sigma_{-j}^{(1)}>x-h(x)\right) & =\int_{0}^{h(x)} \mathbf{P}\left(Q_{j}^{(1)} \in d t\right) \mathbf{P}\left(\sigma_{-j}^{(1)}>x-t\right) \\
& +\mathbf{P}\left(Q_{j}^{(1)}>h(x)\right) \mathbf{P}\left(\sigma_{-j}^{(1)}>x-h(x)\right)
\end{aligned}
$$

where

$$
\mathbf{P}\left(Q_{j}^{(1)}>h(x)\right) \leq \mathbf{P}(Z>h(x))
$$


and

$$
\mathbf{P}\left(\sigma_{-j}^{(1)}>x-h(x)\right) \sim c^{(1)} \bar{F}(x-h(x)) \sim c^{(1)} e^{\gamma h(x)} \bar{F}(x) .
$$

Since $\mathbf{E} e^{\gamma Z}<\infty$, we get $\mathbf{P}(Z>h(x)) e^{\gamma h(x)} \rightarrow 0$ when $x \rightarrow \infty$. Therefore, the second equality also holds. The third equality follows from the uniform equivalence (66) and from the assumptions of the theorem:

$\int_{0}^{h(x)} \mathbf{P}\left(Q_{j}^{(1)} \in d t\right) \mathbf{P}\left(\sigma_{-j}^{(1)}>x-t\right) \sim c^{(1)} \int_{0}^{h(x)} \mathbf{P}\left(Q_{j}^{(1)} \in d t\right) \bar{F}(x-t) \sim c^{(1)} \int_{0}^{h(x)} \mathbf{P}\left(Q_{j}^{(1)} \in d t\right) e^{\gamma t} \bar{F}(x)$.

Finally, as $x \rightarrow \infty$,

$$
\int_{0}^{h(x)} \mathbf{P}\left(Q_{j}^{(1)} \in d t\right) e^{\gamma t} \rightarrow \int_{0}^{\infty} \mathbf{P}\left(Q_{j}^{(1)} \in d t\right) e^{\gamma t}=\mathbf{E} e^{\gamma Q_{j}^{(1)}}
$$

and the last equality follows from the mutual independence of the summands in $Q_{j}^{(1)}$.

Hence,

$$
\sum_{j=0}^{N L} \mathbf{P}\left(Z>x, \sigma_{-j}^{(1)}>x-h(x)\right)=(1+o(1)) c^{(1)} \bar{F}(x) \mathbf{E} e^{\gamma W_{0}^{(1)}} \sum_{j=0}^{N L} \mathbf{E} e^{\gamma Y_{j}^{(1)}}\left(\varphi_{\tau}(-\gamma)\right)^{j}+o(\mathbf{P}(Z>x)) .
$$

Similarly, for any $j=0,1,2, \ldots$, the random variable $Z$ may be represented as

$$
Z=\max \left(V_{J}^{(2)}, Y_{j}^{(2)}+\sigma_{-j}^{(2)}+\sum_{i=-j+1}^{0} \sigma_{i}^{(2)}-\sum_{i=-j}^{-1} \tau_{i}\right)
$$

where

$$
Y_{j}^{(2)}=\sup _{m \geq n \geq-j}\left(\sum_{-m}^{-n} \sigma_{i}^{(1)}+\sum_{-n}^{-j-1} \sigma_{i}^{(2)}-\sum_{-m}^{-j-1} \tau_{i}\right)
$$

(and the distribution of $Y_{j}^{(2)}$ does not depend on $j$ ),

$$
V_{j}^{(2)}=\sup _{m \geq-j} \max _{0 \leq n<-j}\left(\sum_{-m}^{-n} \sigma_{i}^{(1)}+\sum_{-n}^{0} \sigma_{i}^{(2)}-\sum_{-m}^{-1} \tau_{i}\right)
$$

and random variables $\left(Y_{j}^{(2)}, \sum_{i=-j+1}^{0} \sigma_{i}^{(2)}-\sum_{i=-j}^{-1} \tau_{i}, V_{j}^{(2)}\right)$ are mutually independent of $\sigma_{-j}^{(2)}$. Then $\left(\right.$ with $Q_{j}^{(2)}=Y_{j}^{(2)}+\sum_{i=-j+1}^{0} \sigma_{i}^{(2)}-\sum_{i=-j}^{-1} \tau_{i}$ )

$$
\begin{aligned}
\mathbf{P}\left(Z>x, \sigma_{-j}^{(2)}>x-h(x)\right) & =\mathbf{P}\left(Q_{j}^{(2)}+\sigma_{-j}^{(2)}>x, \sigma_{-j}^{(2)}>x-h(x)\right)+o(\mathbf{P}(Z>x)) \\
& =\int_{0}^{h(x)} \mathbf{P}\left(Q_{j}^{(2)} \in d t\right) \mathbf{P}\left(\sigma_{-j}^{(2)}>x-t\right)+o(\mathbf{P}(Z>x)+\bar{F}(x)) \\
& =c^{(2)} \bar{F}(x) \int_{0}^{h(x)} \mathbf{P}\left(Q_{j}^{(2)} \in d t\right) e^{\gamma t}+o(\mathbf{P}(Z>x)+\bar{F}(x)) \\
& =c^{(2)} \bar{F}(x) \mathbf{E} e^{\gamma Y_{0}^{(2)}}\left(\varphi_{(2)}(\gamma)\right)^{j-1}\left(\varphi_{\tau}(-\gamma)\right)^{j-1}+o(\mathbf{P}(Z>x)+\bar{F}(x))
\end{aligned}
$$

(where the arguments are similar to those which were used to obtain the asymptotics for $\left.\mathbf{P}\left(Z>x, \sigma_{-j}^{(2)}>x-h(x)\right)\right)$. Thus,

$$
\sum_{j=0}^{N L} \mathbf{P}\left(Z>x, \sigma_{-j}^{(2)}>x-h(x)\right)=(1+o(1)) c^{(2)} \bar{F}(x) \mathbf{E} e^{\gamma Y_{0}^{(2)}} \frac{1-R_{2}^{N L}}{1-R_{2}}+o(\mathbf{P}(Z>x))
$$


where $R_{2}=\varphi_{(2)}(\gamma) \varphi_{\tau}(-\gamma)<1$.

Putting together (22), (23) and (24), we get:

$$
\begin{aligned}
\mathbf{P}(Z>x)(1+o(1)) & =(1+o(1)) \bar{F}(x) \\
& \times\left(c^{(1)} \mathbf{E} e^{\gamma W_{0}^{(1)}} \sum_{j=0}^{N L} \mathbf{E} e^{\gamma Y_{j}^{(1)}}\left(\varphi_{\tau}(-\gamma)\right)^{j}+c^{(2)} \mathbf{E} e^{\gamma Y_{0}^{(2)}} \frac{1-R_{2}^{N L}}{1-R_{2}}\right)+g_{3}(x) .
\end{aligned}
$$

Therefore,

$$
\limsup _{x \rightarrow \infty} \frac{\mathbf{P}(Z>x)}{\bar{F}(x)} \leq c^{(1)} \mathbf{E} e^{\gamma W_{0}^{(1)}} \sum_{j=0}^{\infty} \mathbf{E} e^{\gamma Y_{j}^{(1)}}\left(\varphi_{\tau}(-\gamma)\right)^{j}+c^{(2)} \mathbf{E} e^{\gamma Y_{0}^{(2)}} \frac{1}{1-R_{2}}+\varepsilon
$$

and

$$
\liminf _{x \rightarrow \infty} \frac{\mathbf{P}(Z>x)}{\bar{F}(x)} \geq c^{(1)} \mathbf{E} e^{\gamma W_{0}^{(1)}} \sum_{j=0}^{N L} \mathbf{E} e^{\gamma Y_{j}^{(1)}}\left(\varphi_{\tau}(-\gamma)\right)^{j}+c^{(2)} \mathbf{E} e^{\gamma Y_{0}^{(2)}} \frac{1-R_{2}^{N L}}{1-R_{2}}
$$

for any positive $\varepsilon$ (and, respectively, for any positive integer $N$ ). Letting $\varepsilon$ to zero, we obtain finally:

$$
\mathbf{P}(Z>x) \sim \bar{F}(x)\left(c^{(1)} \mathbf{E} e^{\gamma W_{0}^{(1)}} \sum_{j=0}^{\infty} \mathbf{E} e^{\gamma Y_{j}^{(1)}}\left(\varphi_{\tau}(-\gamma)\right)^{j}+c^{(2)} \mathbf{E} e^{\gamma Y_{0}^{(2)}} \frac{1}{1-R_{2}}\right) .
$$

We prove now the bounds (10). For this, we use repeatedly the following relations: for any finite or countable collection of random variables $X_{i}$,

$$
\sup _{i} \mathbf{E} e^{X_{i}} \leq \mathbf{E} e^{\sup _{i} X_{i}} \leq \sum_{i} \mathbf{E} e^{X_{i}}
$$

First,

$$
1 \leq \mathbf{E} e^{\gamma W_{0}^{(1)}} \leq \sum_{n=0}^{\infty}\left(\mathbf{E} e^{\gamma\left(\sigma_{1}^{(1)}-\tau_{1}\right)}\right)^{n}=\frac{1}{1-R_{1}}
$$

where again $R_{1}=\varphi_{(1)}(\gamma) \varphi_{\tau}(-\gamma)<1$. Further, with $\varphi(\gamma)=\max \left(\varphi_{(1)}(\gamma), \varphi_{(2)}(\gamma)\right)$,

$$
\begin{aligned}
\mathbf{E} e^{\gamma Y_{j}^{(1)}} & \geq \max _{0 \leq k \leq-j} \mathbf{E} \exp \left(\gamma \sum_{i=-j+1}^{-j+k} \sigma_{i}^{(1)}+\gamma \sum_{i=-j+k}^{0} \sigma_{i}^{(2)}\right) \\
& =\max _{0 \leq k \leq-j} \varphi_{(1)}^{k}(\gamma) \varphi_{(2)}^{j+1-k}(\gamma)=\varphi^{j}(\gamma) \varphi_{(2)}(\gamma)
\end{aligned}
$$

and

$$
\begin{aligned}
\mathbf{E} e^{\gamma Y_{j}^{(1)}} & \leq \sum_{k=0}^{-j} \mathbf{E} \exp \left(\gamma \sum_{i=-j+1}^{-j+k} \sigma_{i}^{(1)}+\gamma \sum_{i=-j+k}^{0} \sigma_{i}^{(2)}\right) \\
& =\varphi_{(2)}(\gamma) \sum_{i=0}^{j} \varphi_{(1)}^{i}(\gamma) \varphi_{(2)}^{j-i}(\gamma) .
\end{aligned}
$$

Since $R=\varphi(\gamma) \varphi_{\tau}(-\gamma)$,

$$
\frac{\varphi_{(2)}(\gamma)}{1-R} \leq \sum_{j=0}^{\infty} \mathbf{E} e^{\gamma Y_{j}^{(1)}} \varphi_{\tau}(-\gamma)^{j} \leq \varphi_{(2)}(\gamma) \cdot \frac{1}{\left(1-R_{1}\right)\left(1-R_{2}\right)}
$$

Similarly,

$$
\varphi_{(1)}(\gamma) \leq \mathbf{E} e^{\gamma Y_{0}^{(2)}} \leq \varphi_{(1)}(\gamma) \cdot \frac{1}{\left(1-R_{1}\right)\left(1-R_{2}\right)} .
$$

Substituting all obtained inequalities into (25), we arrive at (10). 


\section{Appendix}

\subsection{Properties of distributions from the class $\mathcal{S}_{\beta}, \beta>0$.}

We present here a number of known properties (Properties 1-3) of the class $\mathcal{S}_{\beta}$ with $\beta>0$ - see, for example, [11] and the comments in [5], and also Property 4 which follows from results of [5].

Property 1. (Closure of the class $\mathcal{S}_{\beta}$ with respect to the tail equivalence)

If $F \in \mathcal{S}_{\beta}$ and $\bar{F}(x) \sim c \bar{G}(x)$ for some constant $c \in(0, \infty)$, then $G \in \mathcal{S}_{\beta}$. If, in particular, random variables $X$ and $Y$ are independent, $Y$ is a.s. non-negative and. $F(x)=\mathbf{P}(X \leq$ $x) \in \mathcal{S}_{\beta}$, then

$$
\bar{G}(x)=\mathbf{P}(X-Y>x) \sim \int_{0}^{h(x)} G(d t) \bar{F}(x+t) \sim \bar{F}(x) \int_{0}^{h(x)} G(d t) e^{-\beta t} \sim \bar{F}(x) \mathbf{E} e^{-\beta Y}
$$

as $x \rightarrow \infty$ and, therefore, $G \in \mathcal{S}_{\beta}$.

The following more general result also holds.

Property 2. Assume that $F \in \mathcal{S}_{\beta}$ for some $\beta \geq 0$. Assume also that random variables $X_{i}, i=1, \ldots, n$ are mutually independent and their distribution functions $F_{i}$ satisfy the relations $\mathbf{P}\left(X_{i}>x\right)=\overline{F_{i}}(x) \sim c_{i} \bar{F}(x)$ as $x \rightarrow \infty$, for some $c_{i} \geq 0, \sum_{1}^{n} c^{(i)}>0$. Then $\varphi_{i}=\mathbf{E} e^{\beta X_{i}}<\infty$ for all $i=1, \ldots, n$. The distribution of the sum $\sum_{i=1}^{n} X_{i}$ also belongs to the class $\mathcal{S}_{\beta}$ and

$$
\begin{aligned}
\mathbf{P}\left(\sum_{i=1}^{n} X_{i}>x\right) & \sim \sum_{j=1}^{n} \mathbf{P}\left(\bigcup_{j=1}^{n}\left\{\sum_{i \neq j} X_{i} \leq h(x), \sum_{i=1}^{n} X_{i}>x\right\}\right) \\
& \sim \sum_{j=1}^{n} \mathbf{P}\left(\sum_{i \neq j} X_{i} \leq h(x), \sum_{i=1}^{n} X_{i}>x\right) \\
& \sim \sum_{j=1}^{n} \mathbf{P}\left(\bigcup_{j=1}^{n}\left\{X_{j}>x-h(x), \sum_{i=1}^{n} X_{i}>x\right\}\right) \\
& \sim \sum_{j=1}^{n} \mathbf{P}\left(X_{j}>x-h(x), \sum_{i=1}^{n} X_{i}>x\right) \sim \prod_{i=1}^{n} \varphi_{i} \sum_{i=1}^{n} \frac{c_{i}}{\varphi_{i}} \bar{F}(x)
\end{aligned}
$$

where $h(x)$ is any function satisfying (6) .

Property 3. Assume that $F \in \mathcal{S}_{\beta}$ for some $\beta \geq 0$. Assume that random variables $V, \xi$ and $\eta$ are mutually independent, $\eta \geq 0$ a.s., $\mathbf{P}(V>x) \sim c_{1} \bar{F}(x)$ and $\mathbf{P}(\xi>x) \sim c_{2} \bar{F}(x)$ where $c_{1} \geq 0$ and $c_{2}>0$. Then, for any function $h$ satisfying (6),

$$
\begin{aligned}
\mathbf{P}(V+\xi-\eta>x, V \leq h(x)) & \sim \mathbf{P}(V+\xi-\eta>x, V-\eta \leq h(x)) \\
& \sim \mathbf{P}(V+\xi-\eta>x, \xi-\eta \geq x-h(x)) \\
& \sim \mathbf{P}(V+\xi-\eta>x, \xi \geq x-h(x)) \\
& \sim c_{2} \bar{F}(x) \mathbf{E} e^{\beta V} \mathbf{E} e^{-\beta \eta} .
\end{aligned}
$$

Property 4. Consider a sequence of i.i.d. random variables $\left\{X_{n}\right\}$ with a common distribution function $F$ and assume that $\mathbf{E} X_{i}=-a<0, F \in \mathcal{S}_{\beta}$ and $\mathbf{E} e^{\beta X_{1}}<1$. Let $M_{k}=$ $\max _{0 \leq n \leq k} \sum_{i=1}^{n} X_{i}$ and $M=\sup _{n \geq 0} \sum_{i=1}^{n} X_{i}$. It follows from [5, 1 and Remark 3] that

$$
\lim _{x \rightarrow \infty} \frac{\mathbf{P}(M>x)}{\bar{F}(x)}=\frac{\mathbf{E} e^{\beta M}}{1-\mathbf{E} e^{\beta X_{1}}}
$$


(see also [12]) and, moreover, for any (as small as possible) $\varepsilon \in(0,1)$, there exists a sufficiently large $N$ such that, as $x \rightarrow \infty$ and for any function $h(x)$ satisfying (6),

$$
\begin{aligned}
\mathbf{P}(M>x) & \geq \mathbf{P}\left(M>x, \bigcup_{n=1}^{N}\left\{X_{n}>x-h(x)\right\}\right)+o(\bar{F}(x)) \\
& =\sum_{n=1}^{N} \mathbf{P}\left(M>x, X_{n}>x-h(x)\right)+o(\bar{F}(x)) \\
& \geq \mathbf{P}(M>x)+o(\bar{F}(x))-\varepsilon \bar{F}(x)
\end{aligned}
$$

(recall that the notation $f(x) \geq g(x)+o(f(x))$ means $\lim _{\sup _{x \rightarrow \infty}} g(x) / f(x) \leq 1$; in our case $o(\bar{F}(x))=o(\mathbf{P}(M>x)))$.

If we assume in addition that the random variables $X_{n}$ are represented as differences $X_{n}=\xi_{n}-\eta_{n}$ where $\left\{\xi_{n}\right\}$ and $\left\{\eta_{n}\right\}$ are two mutually independent sequences of i.i.d. random variables and random variables $\xi_{n}$ are non-negative a.s., then the relations above stay valid if we replace the events $\left\{X_{n}>x-h(x)\right\}$ by $\left\{\xi_{n}>x-h(x)\right\}$.

\subsection{A simple proof of Theorem 1 by the use of upper and lower bounds from Subsection 2.1.}

Recall again that the stationary waiting time in a single-server queue with service times $\sigma_{n}$ and inter-arrival times $\tau_{n}$ has the same distribution as the supremum $M=\sup _{n} S_{n}$ of a random walk $S_{n}=\sum_{1}^{n} X_{i}$ with increments $X_{n}=\sigma_{n}-\tau_{n}$. Apply the following well-known result for a random walk $S_{n}=\sum_{i=1}^{n} X_{i}$ with negative drift:

Theorem 3. If

$$
\lambda_{0}=\sup \left\{\lambda: \varphi_{X_{1}}(\lambda) \leq 1\right\}>0,
$$

then

$$
-\ln \mathbf{P}(M>x) \sim \lambda_{0} x .
$$

Remark 8. We are aware of only one publication ([8, p. 17) where Theorem 3 is formulated without extra assumptions. Usually authors assume in addition (see, for example, [10, Section 21, Theorem 11]) the so-called Cramer condition

$$
\varphi_{X_{1}}\left(\lambda_{0}\right)=1 \quad \text { and } \quad d=\mathbf{E} X_{1} e^{\lambda_{0} X_{1}}<\infty
$$

or even stronger conditions. The theorem may be obtained also as a corollary of more general results, for instance, from [10]. We provide in Subsection 3.3 a methodological comment on how one can prove the general result of Theorem 3 given that it is already proven under the conditions (26).

It follows from Theorem 3 that

$$
-\ln \mathbf{P}\left(W^{(i)}>x\right) \sim \gamma^{(i)} x
$$

and since $Z^{(i)} \geq W^{(i)}$ a.s.,

$$
\limsup _{x \rightarrow \infty} \frac{-\ln \mathbf{P}(Z>x)}{x} \leq \gamma
$$


On the other hand, for any $\lambda>0$,

$$
\begin{aligned}
\widetilde{\varphi}(\lambda) & \equiv \mathbf{E} e^{\lambda \widetilde{\sigma}_{1}} \leq \sum_{j=1}^{L} \mathbf{E} \exp \left(\sum_{i=1}^{j} \sigma_{i}^{(1)}+\sum_{i=j}^{L} \sigma_{i}^{(2)}\right) \\
& =\sum_{j=1}^{L} \varphi_{(1)}^{j}(\lambda) \varphi_{(2)}^{L-j+1}(\lambda) \equiv \varphi_{*}(\lambda) .
\end{aligned}
$$

Using again the notation $\varphi(\lambda)=\max \left(\varphi_{(1)}(\lambda), \varphi_{(2)}(\lambda)\right)$, we get

$$
\min \left(\left(\varphi_{(1)}(\lambda), \varphi_{(2)}(\lambda)\right) \cdot \varphi^{L}(\lambda) \leq \widetilde{\varphi}(\lambda) \leq \varphi_{*}(\lambda) \leq(L+1) \varphi^{L+1}(\lambda)\right.
$$

and therefore

$$
(\widetilde{\varphi}(\lambda))^{1 / L} \rightarrow \varphi(\lambda) \quad \text { and } \quad\left(\varphi_{*}(\lambda)\right)^{1 / L} \rightarrow \varphi(\lambda) \quad \text { as } \quad L \rightarrow \infty .
$$

Let $\widetilde{\gamma}=\sup \left\{\lambda: \widetilde{\varphi}(\lambda) \varphi_{\tau}^{L}(-\lambda) \leq 1\right\}$. Since $\widetilde{\sigma}_{1} \geq \max \left(\sum_{1}^{L} \sigma_{i}^{(1)}, \sum_{1}^{L} \sigma_{i}^{(2)}\right)$, we may conclude that $\gamma \geq \widetilde{\gamma}$. $>$ From (28), $\widetilde{\gamma} \rightarrow \gamma$. By Theorem 3, for any sufficiently large $L$,

$$
-\ln \mathbf{P}(\widetilde{W}>x) \sim \widetilde{\gamma} x
$$

$>$ From $\mathbf{E} \exp \left(\gamma \widetilde{\sigma}_{0}\right)<\infty$, we get

$$
-\ln \mathbf{P}(\widetilde{Z}>x) \sim \widetilde{\gamma} x
$$

Letting $L$ to infinity, we obtain

$$
\liminf _{x \rightarrow \infty} \frac{-\ln \mathbf{P}(Z>x)}{x} \geq \gamma .
$$

The statement of Theorem 1 follows now from the inequalities (27) and (29).

Remark 9. A natural analogue of Theorem 1 holds for a tandem of any finite number of queues, with a similar proof.

\subsection{A comment on a proof of Theorem 3 .}

Assume that the statement of the theorem has been already proved under the additional assumptions (26). Note that there are several versions of such a proof (by the use of, say, (a) martingale techniques, (b) exponential change of measure and elements of the renewal theory, etc.)

Assume now that conditions (26) do not hold. For any $r>0$, define random variables

$$
X_{n,+r}=\max \left(X_{n},-r\right) \quad \text { and } \quad X_{n,-r}=\min \left(X_{n}, r\right) .
$$

Denote the corresponding sums, maxima and moments by $S_{n,+r}, S_{n,-r}, M_{+r}, M_{-r}, \varphi_{X,+r}$ and $\varphi_{X,-r}$ where $M_{+r} \geq M \geq M_{-r}$ a.s. For all sufficiently large values of $r$, the maximum $M_{+r}$ is a.s. finite. From the monotonicity and continuity of $\varphi_{X,+r}$ and $\varphi_{X,-r}$ as functions of $r$ and from the boundedness from above of random variables $X_{n,-r}$, it follows, firstly, that the roots $\lambda_{+r}<\lambda_{0}<\lambda_{-r}$ of equations $\varphi_{X,+r}\left(\lambda_{+r}\right)=1$ and $\varphi_{X,-r}\left(\lambda_{-r}\right)=1$ exist for any $r$, the corresponding derivatives are finite and therefore

$$
-\ln \mathbf{P}\left(M_{+r}>x\right) \sim \lambda_{+r} x \quad \text { and } \quad-\ln \mathbf{P}\left(M_{-r}>x\right) \sim \lambda_{-r} x,
$$


and, second, both $\lambda_{+r}$ and $\lambda_{-r}$ converge to $\lambda_{0}$ as $r \rightarrow \infty$.

If $\varphi_{X}\left(\lambda_{0}\right)<1$, then there is $r<\infty$ such that $\varphi_{X,+r}\left(\lambda_{0}\right)=1$ and $\varphi_{X,+r}^{\prime}\left(\lambda_{0}\right)<\infty$. Then, for this $r$,

$$
-\ln \mathbf{P}\left(M_{+r}>x\right) \sim \lambda_{0} x,
$$

and the statement of Theorem 3 follows.

The case $\varphi_{X}\left(\lambda_{0}\right)=1$ and $d=\infty$ is left for a reader.

The author would like to thank Stan Zachary for improving the style of the English translation, and the referee for a number of important comments and remarks.

Remark (added at the proofreading): in the paper [13], the author develops the approach from [7] to obtain the logarithmic asymptotics in a wide class of stochastic networks. 


\section{Список литературы}

[1] Loynes, R. M. "The stability of a system of queues in series," Math. Proc. Cambridge Phil. Soc., 1964, vol. 60, no.3, pp. 569-574.

[2] Baccelli, F. and Foss, S. "Ergodicity of Jackson-Type Queueing Networks", Queueing Systems, 1994, vol. 17, no. 1, pp. 5-72.

[3] Bertsimas, D., Paschalidis, I., and Tsitsiklis, J. "On the large deviation behaviour in acyclic networks of G/G/1 queues", Ann. Appl. Prob., 1998, vol. 8, no. 4, pp. $1027-1069$

[4] Ganesh, A. J. "Large deviations of the sojourn time for queues in series", Ann. Oper. Res., 1998, vol. 79, no. 1, pp. 3-26.

[5] Zachary, S. and Foss, S. G. "On the exact distributional asymptotics for the suprumem of a random walk with increments in a class of light-tailed distributions", Siberian Math. J., 2006, v. 47 , np. 6, pp. 1034-1041.

[6] Baccelli, F. and Foss, S. "Moments and tails in monotone-separable stochastic networks", Ann. Appl. Prob, 2004, vol. 14, no. 3, pp. 612-650.

[7] Baccelli, F. and Foss, S. "On the Saturation Rule for the Stability of Queues". J. Appl. Prob., 1995, vol. 32, no. 2, pp. 494-507

[8] Ganesh, A. J., O Connell, N., Wischik, D. "Big queues", Lecture Notes in Mathematics, vol. 1838, Springer, 2004.

[9] Borovkov, A. A. "Stochastic Processes in Queueing Theory". Wiley, New York, 1976.

[10] Borovkov, A. A. and Mogul'skii A. A. "The second deviations function and the asymptotic problems of renewal and hitting the boundary for multidimensional random walks" Siberian Math. J., 1996, v. 37, no. 4, pp. 647-682.

[11] Pakes, A. "On the tails of waiting time distributions." J. Appl. Prob., 1975, vol. 7, no.4, pp. $745-789$.

[12] Bertoin, J. and Doney, R. A. "Some asymptotic results for transient random walks", Adv. Appl. Prob., 1996, vol. 28, no.1, pp. 207-226.

[13] Lelarge, M. "Tail asymptotics for monotone-separable networks", J. Appl. Prob., 2007, vol. 44 , no. 2 , pp. $306-320$. 